\title{
Non-Endoscopic and Endoscopic Adhesiolysis in Post Lumbar Laminectomy Syndrome: A One-Year Outcome Study and Cost Effectiveness Analysis
}

\author{
Laxmaiah Manchikanti, M.D., Vidyasagar Pampati, M. Sc., Cyrus E. Bakhit, M.D., and \\ Rajgopal R. Pakanati, M.D.
}

Pain Management Center of Paducah, Paducah, KY

\begin{abstract}
Post lumbar laminectomy syndrome with its resultant chronic low back pain is estimated to occur in $20 \%$ to $50 \%$ of the patients. Among various procedures available, lysis of epidural adhesions is considered as one of the effective therapeutic modalities of management in these patients, and may be performed either non-endoscopically or endoscopically.
\end{abstract}

This retrospective evaluation included 120 post lumbar laminectomy patients who underwent either non-endoscopic adhesiolysis (Group I) or endoscopic adhesiolysis (Group II) with 60 consecutive patients in each group. The quality of pain relief when greater than $50 \%$ was considered significant. Results showed all patients experienced significant relief following both procedures even though the number of patients experiencing significant relief decreased with both techniques over a time period. Overall relief with the first procedure (mean + SEM) was $12+$ 3.2 weeks for Group I, and it was $20+2.9$ weeks for Group II with significantly longer improvement in Group II than Group I. At one year follow up, the results showed that with repeat procedures, $72 \%$ in Group I and $40 \%$ in Group II experienced significant relief at 6 months, whereas at 12 months, it decreased

Low back pain secondary to post lumbar laminectomy syndrome, also known as failed back surgery syndrome, or failed management syndrome is a growing entity in modern medicine (1-19). An estimated 20\% to 50\% of lumbar surgeries result in failed back surgery syndrome either from surgery that was inadequate, incorrect, or unnecessary, even though this syndrome often results following a well indicated and well performed surgical intervention. Fager and Freidberg (12) analyzed the failure and poor results of lumbar spine surgery and concluded that $51 \%$ of the patients had more than one operation and only $32 \%$ improved following the initial operation. Fritsch et al (8) reported that in $80 \%$ of the patients results were satisfactory in short-term evaluation, decreasing to $22 \%$ in long-term follow-up after lumbar surgical intervention $(1,2,6,12,13,19)$. Address correspondence: Laxmaiah Manchikanti, M.D., Pain Management Center of Paducah, 2831 Lone Oak Road, Paducah, KY 42003 to $52 \%$ in Group I and $22 \%$ in Group II, with a significantly greater number of patients experiencing relief at 6 months and 12 months in Group I, than Group II, even though Group I patients underwent a greater number of procedures.

Cost effectiveness analysis showed Group I patients experiencing significant relief at a cost of $\$ 40$ per week, with one year quality of life improvement for $\$ 2,080$, whereas it was $\$ 135$ per week improvement in Group II with a one year quality of life improvement at a cost of $\$ 7,020$ with significant difference noted in cost effectiveness.

In conclusion, non-endoscopic epidural adhesiolysis and administration of corticosteroids and hypertonic saline is a safe and cost effective procedure for relieving chronic intractable pain in post lumbar laminectomy patients who failed to respond to other modalities of treatment. Similarly, endoscopic adhesiolysis with the administration of corticosteroids is also a safe and possibly cost-effective technique for relief of chronic intractable pain failing to respond to other modalities of treatments.

Keywords: Chronic Low Back Pain, Epidural Fibrosis, Adhesiolysis

Waddell et al (6) studied 103 patients who failed initial surgery, documenting the declining success of subsequent surgical interventions. The causes of failed back syndrome are epidural fibrosis, arachnoiditis, neural encroachment, and mechanical instability $(16,18,21)$. It is largely agreed upon that epidural fibrosis presents a considerable amount of morbidity in lumbar surgery (16-18). Further surgery, even with extensive evaluation and individualization based on the primary pathology resulted in success rates as low as $12 \%(18,21)$.

Ross et al (7) in the study of the relationship between peridural scar evaluated by Magnetic Resonance Imaging and recurrent radicular pain after lumbar discectomy showed that subjects with extensive peridural scarring were 3.2 times more likely to experience recurrent radicular pain. Even modern developments in spinal surgery with microsurgical approaches have added new categories of treat- 
ment failure (18). Analysis of the frequency and location of lumbar and ventral dural adhesions in elderly cadavers showed significant evidence of adhesions in $40 \%$ at L4/5 levels, in 36\% at L5/S1 levels, and in 16\% at L3/4 levels (9). Arachnoiditis and epidural fibrosis were relatively rare entities before the introduction of lumbar spinal surgery for degenerative conditions. Numerous reports where epidural fibrosis was found on repeat surgery after the first surgery was unsuccessful, led to the speculation of associating recurrent symptoms with scarring $(1-4,7-13,19)$. The search for inhibition of epidural scar formation after lumbar laminectomy has been largely unsuccessful (21). The effectiveness of a large variety of therapeutic interventions available for the management of post lumbar laminectomy pain syndrome, which has not been studied extensively, as well as low back pain in general, has not been demonstrated conclusively, resulting in an inordinate challenge to the clinician. Among various procedures available, lysis of epidural adhesions is considered as one of the effective therapeutic modalities of management in these patients. Epidural adhesiolysis is performed either non-endoscopically or endoscopically (22-31).

Non-endoscopic adhesiolysis is performed with a Racz ${ }^{\circledR}$ epidural tunnel catheter which is a stainless steel, Teflon coated, soft, spiral tipped catheter, presumably passing this into the area of adhesions or the scar on multiple occasions, thus, achieving adhesiolysis (22-28). In contrast, endoscopic adhesiolysis involves accessing the epidural space using a flexible fiberoptic catheter via the sacral hiatus, thus obtaining three-dimensional visualization of the contents, and with the ability to steer the catheter toward structures of interest, namely adhesions (29-31). With a non-endoscopic procedure, adhesions are identified as filling defects and subsequent filling of these areas is considered as achieving satisfactory adhesiolysis. However, with endoscopy, the video-images allow examination of a specific nerve root and its pathology. With both techniques, the purpose is to accurately place the injectate intended for delivery in the epidural space and onto the nerve root. Both techniques have various advantages and disadvantages which include complications and costs. However, safety and cost effectiveness of either technique has not been evaluated in post lumbar laminectomy syndrome. The purpose of this evaluation is to estimate the value and safety of non-endoscopic, as well as endoscopic adhesiolysis in managing chronic low back pain following post lumbar laminectomy syndrome non-responsive to other modalities of conservative management.

\section{METHODS}

This retrospective evaluation included 120 post lumbar laminectomy patients who underwent either non-endo- scopic adhesiolysis or endoscopic adhesiolysis. Group I consisted of 60 consecutive patients who underwent nonendoscopic adhesiolysis during 1997, while Group II consisted of 60 consecutive patients undergoing endoscopic adhesiolysis during 1998. Inclusion criteria was to have had one or more surgical interventions, and exclusion of facet joint pain, or sacroiliac joint pain as the major causative factors of pain and disability. All other modalities of treatment failed to provide substantial relief of at least 6 weeks or longer with a single treatment which included epidural injections, physical therapy, and drug therapy. This survey provided 18 to 32 months of interval between the treatment and evaluation. Evaluation included patient characteristics of age, gender, duration of pain in years, mode of onset of the pain, and types of surgery. The number of procedures performed on each patient and pain relief in each group was noted up to at least one year. The quality of pain relief with each procedure was characterized as no relief, less than $50 \%$ relief, and greater than $50 \%$ relief. Pain relief greater than $50 \%$ was considered significant and these patients were characterized as "successful with significant pain relief" for each procedure at less than 1 month, 1 month, 2 months, 3 months, 6 months, and 12 months.

All the procedures were performed under fluoroscopy in an ambulatory surgery setting in a sterile operating room by one physician. The technique for adhesiolysis utilizing a catheter included access to the epidural space utilizing $\mathrm{RK}^{\circledR}$ needle (Epimed International Inc, Gloverville, NY), followed by an epidurogram identifying the filling defects and/or epidural fibrosis. Adhesiolysis in Group I was carried out utilizing a Racz ${ }^{\circledR}$ catheter (Epimed International Inc, Gloverville, NY) with final positioning of the catheter to the site of the defect and the source of the pain and an additional injection of contrast to identify successful adhesiolysis followed by injection of 5 cc of Xylocaine ${ }^{\circledR}, 1 \%$ preservative free and $6 \mathrm{mg}$ of Celestone ${ }^{\circledR}$ Soluspan $^{\circledR}$ followed by the injection of $10 \%$ sodium chloride solution in two divided doses of $3 \mathrm{cc}$ each over a period of 10 to 15 minutes.

Adhesiolysis with epiduroscopy was also performed in the operating room under fluoroscopic visualization with entry into the epidural space through the sacral hiatus with a \#16 RK ${ }^{\circledR}$ needle followed by insertion of a guide wire. Subsequently, a dilator and introducer were placed in each patient though a small incision and a fiberoptic endoscope (Myelotec ${ }^{\circledR}$, Roswell, GA) was introduced into the epidural space through the introducer, obtaining video images. Gentle irrigation with normal saline was utilized to distend the epidural space. After visualization of the nerve roots and reproduction of the pain, adhesiolysis was carried out in each patient followed by an injection of $10 \mathrm{cc}$ of 
Xylocaine $^{\circledR}, 1 \%$ preservative free mixed with $6 \mathrm{mg}$ of Celestone $^{\circledR}$ Soluspan ${ }^{\circledR}$.

Quality and duration of pain relief and change in functional status, as well as complications were monitored with each follow-up visit.

Statistical analysis was performed utilizing SPSS for Windows, Release 9.0.1, 1999, Chicago: SPSS Inc. Distribution of age, gender, duration of pain, onset of the pain, and type of surgery between groups were tested by chi-squared test. Significant pain relief was tested by utilizing $2 \times 2$ chisquared test used as simple effects follow-up tests. Student "t" test was utilized to compare the means. Differences were considered significant when $\mathrm{P}$ values were less than 0.05 .

\section{RESULTS}

\section{Patient Characteristics}

Follow-up was available in all of the patients. As shown in Table 1, no significant differences were noted either in age distribution, gender distribution, duration of pain, mode of onset of the pain, or type(s) of surgical intervention(s).

\section{Injection Characteristics}

The details of the number of procedures in each group showed that the number of patients undergoing multiple procedures was significantly higher in Group I than in Group II (Table 2). During the period of one year in Group I, 4 patients underwent 6 procedures, 3 patients underwent 5 procedures, and 12 patients underwent 4 procedures, in contrast to Group II with maximum number of procedures of 3 only in one patient. Number of procedures per patient were also significantly different with mean + SEM of $2.98+$ 0.16 and $1.28+0.07$ for Groups I and II respectively.

\section{Pain Relief}

Quality and duration of relief associated with each procedure by members of the groups are illustrated in Table 3 . All patients experienced significant relief. However, the number of patients experiencing significant relief decreased to $72 \%$ and $97 \%$ at 1 month, $25 \%$ and $80 \%$ at 2 months, $10 \%$ and $52 \%$ at 3 months, $7 \%$ and $22 \%$ at 6 months, $5 \%$ and $8 \%$ at 12 months in Group I and II respectively. Significant differences were noted between Group I and Group II with endoscopic adhesiolysis achieving relief in a greater number of patients at 1 month, 3 months, 6 months, and 12 months with first procedure, whereas with the second procedure, significant differences were limited to the 2 month and 3 month periods. Some improvement was noted with the second procedure in both groups. However, significant difference was seen only between the first procedure and second procedure in non-endoscopic adhesiolysis
Table 1. Patient Demographics

\begin{tabular}{|c|c|c|}
\hline & Group I & Group II \\
\hline Number of Patients & 60 & 60 \\
\hline $\begin{array}{l}\text { Age (Years) } \\
\text { Mean + SEM } \\
\text { Range } \\
<65 \\
>65\end{array}$ & $\begin{array}{c}51.8+1.76 \\
21-73 \\
90 \% \\
10 \%\end{array}$ & $\begin{array}{c}48.7+1.61 \\
29-79 \\
78 \% \\
22 \%\end{array}$ \\
\hline $\begin{array}{l}\text { Gender } \\
\text { Male } \\
\text { Female }\end{array}$ & $\begin{array}{l}63 \% \\
37 \%\end{array}$ & $\begin{array}{l}48 \% \\
52 \%\end{array}$ \\
\hline $\begin{array}{l}\text { Duration of Pain } \\
\text { (Years) } \\
\text { Mean + SEM } \\
<4 \\
>4\end{array}$ & $\begin{array}{c}7+0.54 \\
30 \% \\
70 \%\end{array}$ & $\begin{array}{c}8+0.51 \\
22 \% \\
78 \%\end{array}$ \\
\hline $\begin{array}{l}\text { Onset of the Pain } \\
\text { Traumatic } \\
\text { Non-Traumatic }\end{array}$ & $\begin{array}{l}57 \% \\
43 \%\end{array}$ & $\begin{array}{l}65 \% \\
35 \%\end{array}$ \\
\hline $\begin{array}{l}\text { Type of Surgical } \\
\text { Intervention(s) } \\
\text { Non-Fusion } \\
\text { Fusion }\end{array}$ & $\begin{array}{l}63 \% \\
37 \%\end{array}$ & $\begin{array}{l}57 \% \\
43 \%\end{array}$ \\
\hline
\end{tabular}

Group I - Non-Endoscopic Adhesiolysis

Group II - Endoscopic Adhesiolysis

At one-year follow-up, the results showed that while 100\% of the patients experienced relief at 1 month in both groups, with repeat procedures, $90 \%$ in Group I and $75 \%$ in Group II at 3 months, $72 \%$ in Group I and $40 \%$ in Group II at 6 months, and 52\% in Group I and 22\% in Group II experienced significant relief at 12 months (Table 4). This analysis showed that a significantly greater number of patients experienced relief at 6 months and 12 months in Group I,

Table 2. Details of number of procedures and patients undergoing multiple procedures during a period of one year

\begin{tabular}{|c|c|c|c|c|}
\hline \multirow[t]{2}{*}{$\begin{array}{l}\text { No. of } \\
\text { procedures }\end{array}$} & \multicolumn{2}{|c|}{$\begin{array}{l}\text { Number of patients per } \\
\text { each procedure }\end{array}$} & \multicolumn{2}{|c|}{$\begin{array}{c}\text { Number of procedures per each } \\
\text { patient }\end{array}$} \\
\hline & Group I & Group II & Group I & Group II \\
\hline 1 & 60 & 60 & 10 & 44 \\
\hline 2 & $50 *$ & 16 & 12 & 15 \\
\hline 3 & $38 *$ & 1 & 19 & 1 \\
\hline 4 & 19 & - & 12 & - \\
\hline 5 & 7 & - & 3 & - \\
\hline 6 & 4 & - & 4 & - \\
\hline \multicolumn{5}{|l|}{ Procedures } \\
\hline Mean & $2.98^{*}$ & 1.28 & $2.98^{*}$ & 1.28 \\
\hline + SEM & +0.07 & +0.16 & +0.16 & +0.07 \\
\hline
\end{tabular}

* Indicates significant difference between Group I and Group II 
Table 3. Duration of significant pain relief (> 50\%) with each procedure

\begin{tabular}{|c|c|c|c|c|}
\hline & \multicolumn{2}{|c|}{ First procedure } & \multicolumn{2}{|c|}{ Second procedure } \\
\hline Pain Relief & $\begin{array}{l}\text { Group I } \\
(\mathrm{N}=60)\end{array}$ & $\begin{array}{c}\text { Group } \\
\text { II } \\
(\mathrm{N}=60)\end{array}$ & $\begin{array}{l}\text { Group I } \\
(\mathrm{N}=50)\end{array}$ & $\begin{array}{c}\text { Group II } \\
(\mathrm{N}=16)\end{array}$ \\
\hline$<1$ month & $100 \%$ & $100 \%$ & $100 \%$ & $100 \%$ \\
\hline 1 month & $72 \% \#$ & $97 \% *$ & $92 \%$ & $94 \%$ \\
\hline 3 months & $25 \%$ & $80 \% *$ & $46 \%$ & $88 \% *$ \\
\hline 6 months & $10 \%$ & $52 \% *$ & $22 \%$ & $75 \% *$ \\
\hline 12 months & $7 \%$ & $22 \% *$ & $10 \%$ & $25 \%$ \\
\hline $\begin{array}{l}>12 \\
\text { months }\end{array}$ & $5 \%$ & $8 \%$ & $4 \%$ & $0 \%$ \\
\hline $\begin{array}{l}\text { Mean } \\
+ \text { SEM } \\
\text { (weeks) }\end{array}$ & $\begin{array}{l}12 \\
+3.2\end{array}$ & $\begin{array}{l}20 * \\
+2.9\end{array}$ & $\begin{array}{l}13 \\
+2.9\end{array}$ & $\begin{array}{l}20^{*} \\
+3.6\end{array}$ \\
\hline
\end{tabular}

* Indicates significant difference between Group I and Group II

\# Indicates significant difference between first procedure and second procedure in Group I

though they underwent greater number of procedures.

\section{Cost Effectiveness}

The pain relief obtained by all 120 patients following surgical intervention was also noted by the history, considering any pain relief greater than $25 \%$ as significant. The results of previous surgery in these patients showed that 120 patients underwent 262 surgical procedures with a relief (mean + SEM) of 11 months per procedure at a cost of $\$ 21,904$ per procedure with $\$ 476$ cost per one week improvement of quality of life with 1 year improvement of quality of life for $\$ 24,752$.

Cost effectiveness of endoscopic and non-endoscopic adhesiolysis was analyzed by calculating the total cost of all procedures including complications in all patients as shown in Table 5. The number of months with significant relief was calculated as 2678 weeks for Group I and 1545 weeks

Table 4. Percent of patients with significant pain relief (> 50\%) on follow-up of one year

\begin{tabular}{ccc}
\hline Follow-up time & Group I & Group II \\
\hline 1 month & $100 \%$ & $100 \%$ \\
3 months & $90 \%$ & $75 \%$ \\
6 months & $72 \% *$ & $40 \%$ \\
12 months & $52 \% *$ & $22 \%$ \\
\hline
\end{tabular}

* Indicates significant difference between Group I and Group II
Table 5. Analysis of cost effectiveness

\begin{tabular}{lcc}
\hline & Group I & Group II \\
$\begin{array}{l}\text { Number of patients } \\
\text { Total number of procedures }\end{array}$ & 60 & 60 \\
$\begin{array}{l}\text { Number of weeks with significant } \\
\text { pain relief (> 50\%) }\end{array}$ & 2678 & 178 \\
$\begin{array}{l}\text { Significant pain relief }(>50 \%) \text { in } \\
\text { weeks per procedure for all } \\
\text { patients in the study (Mean + }\end{array}$ & $15.1+1.93$ & $20.1 *+2.40$ \\
$\begin{array}{l}\text { SEM) } \\
\begin{array}{l}\text { Expenditure per procedure } \\
\text { (Mean + SEM) }\end{array}\end{array}$ & $\$ 601+13.9$ & $\$ 2702 *+170.6$ \\
$\begin{array}{l}\text { Cost per one week improvement } \\
\text { of quality of life }\end{array}$ & $\$ 40$ & $\$ 135^{*}$ \\
$\begin{array}{l}\text { Cost per one year improvement } \\
\text { of quality of life }\end{array}$ & $\$ 2080$ & $\$ 7020$ \\
\hline$*$ Indicates significant difference & & \\
\hline
\end{tabular}

for Group II. The mean significant relief in weeks for the procedures for all the patients in the study was $15.1+1.93$ weeks for Group I, and 20.1 + 2.40 weeks for Group II with longer improvement noted in Group II compared to Group I. Total expenditures were calculated from net collections, as well as all the patient's expenses for outpatient surgical center and physician fees as incurred for the insurer and/ or to the patient. The total cost per procedure was $\$ 601+$ 13.90 in Group I and \$2,702 + \$170.60 in Group II. Significant relief was provided for Group I patients at a cost of $\$ 40$ per week, whereas, it was $\$ 135$ for Group II. Conversion of these cost figures resulted in a determination of a yearly quality of life improvement in Group I patients of $\$ 2,080$, and $\$ 7,020$ in Group II with significant difference noted in cost effectiveness.

\section{Complications}

None of the patients experienced any reactions to the drugs or post lumbar puncture headache. Rash and itching was noted in 3 patients in each group, weight gain in excess of $5 \mathrm{lbs}$ was noted in 1 patient in Group I, subarachnoid puncture was noted with 4 procedures in Group I and 7 procedures in Group II with subarachnoid blockade seen in 2 patients in Group I and 4 patients in Group II. While infection was suspected in none of the patients in Group I, it was suspected in 8 patients in Group II who were administered with postoperative antibiotics. There were no obvious infections in either group. No arachnoiditis, paralysis, weakness, bladder disturbances, or other serious complications were noted in any of the patients. 


\section{DISCUSSION}

Epidural fibrosis as a causative factor for post lumbar laminectomy syndrome is controversial. Prior to the introduction of lumbar spinal surgery for degenerative conditions, chronic adhesional arachnoiditis was described as chronic spinal meningitis (20). The literature comparing symptomatic and asymptomatic patients after lumbar disc or decompressive surgery showed, by and large, a lack of distinction in earlier studies (20), even though Ross et al (7) in a 1996 study showed a significant association between peridural scarring and recurrent radicular pain after lumbar discectomy utilizing magnetic resonance imaging.

Fager and Freidberg (12) analyzed the failures and poor results of lumbar spine surgery and concluded that $51 \%$ of the patients had more than 1 operation; among them $11 \%$ improved, 34\% did not change, and 55\% worsened. They also showed that only $32 \%$ improved following the initial operation, but the improvement was also short-lived; in $50 \%$ of the patients who did show some improvement, it lasted less than 6 months. Waddell et al (6) studied 103 patients who failed initial surgery, documenting that the success of the second operation was $50 \%$ with an additional $20 \%$ considering themselves worse afterward; while with a third procedure, the success rate was $30 \%$ and $25 \%$ considered themselves worse; whereas after four operations, a $20 \%$ success rate was achieved with $45 \%$ of these patients considering themselves worse. These patients who fail to successfully respond to surgery are not only worse off for having had the surgery, but they now enter the high-cost, high-demand, and highly emotional subset of the low back pain (13). Waddell et al (6) showed that the majority of patients fare very poorly with further surgeries as a great number of patients seem to get worse rather than better, while in some the status remains the same.

Devulder et al (32) reported no correlation between adhesiolysis and pain relief as they noted that the filling defects were confirmed in $88 \%$ of the patients with epidurography; but lysis of adhesions failed to correspond with improvement in pain, which was seen in only $33 \%$ of the patients at 1 month, $13 \%$ at 3 months, and $0 \%$ at 12 months. Devulder et al (32) concluded that epidurography might confirm epidural filling defects, but that a better contrast spread during scar lysis does not guarantee sustained relief. Contentious arguments for and against adhesiolysis have been made. The results of non-endoscopic adhesiolysis have been encouraging and the procedure was shown to be cost effective, as well as safe (26-28). Similar results were presented with endoscopic adhesiolysis even though cost effectiveness was not demonstrated $(30,31)$.

The present study showed that an average 1 year quality of life improvement or 1 year of life gain can be achieved at a total cost of \$2,080 and \$7,020 with non-endoscopic and endoscopic adhesiolysis respectively in patients suffering with chronic low back pain secondary to post lumbar laminectomy syndrome non-responsive to various other modalities of treatments. The cost effectiveness of both the procedures appears to be reasonable, but non-endoscopic adhesiolysis appears to be superior than endoscopic adhesiolysis, even though both techniques are superior to the relief obtained with prior surgical interventions. In a recent study, Mueller-Schwefe et al (33), in evaluating cost effectiveness of intrathecal therapy for pain secondary to failed back surgery syndrome, comparing alternative therapies for achieving a defined outcome, reported the cost of medical management to be $\$ 85,186$ per 5 years, $\$ 17,037$ per year, and $\$ 1,420$ per month. They also showed that intrathecal morphine delivery resulted in lower accumulative 60 month costs of $\$ 82,893$ per five years, $\$ 16,579$ per year, and $\$ 1,382$ per month. It is well known that the cost of an inpatient pain treatment program ranges from $\$ 17,000$ to $\$ 25,000$ (34). Costs of outpatient treatment programs range from $\$ 7,000$ to $\$ 10,000$, and chronic pain patients may incur health care bills in excess of $\$ 20,000$ annually for repetitive and redundant tests and interventions (34). It was concluded that in carefully selected patients, the first time lumbar discectomy for the treatment of herniated intervertebral discs provided 1 year of quality of life at a cost of $\$ 29,200$ compared to conservative treatment, and costs for coronary artery bypass grafting, medical treatment for hypertension, and management of depression were $\$ 73,000$, $\$ 38,000$, and $\$ 11,766$ - $\$ 24,403$ respectively (35-37). In addition, cost effectiveness of non-endoscopic adhesiolysis and hypertonic saline neurolysis was previously shown to be $\$ 5,564$ in a heterogenous group of patients for 1 year quality of life improvement (28). Hence, it appears that non-endoscopic, as well as endoscopic adhesiolysis in post lumbar laminectomy patients non-responsive to other modalities of treatments are relatively cost effective.

Similar to all retrospective studies, the current study may be criticized for its retrospective nature, lack of third party interview, and lack of outcome parameters to include return-to-work. Prospective, randomized, double-blind studies are considered as the hallmark of the clinical efficacy of a procedure. However, it is an extremely difficult task as neither physicians nor patients are willing to be randomized to the presumed "control group," in spite of recent reports of surgery to evaluate intracranial procedures. In addition, it is also difficult if not economically impossible, not to mention issues of ethical practice, to simulate either type of adhesiolysis and placebo administration of drugs in a control group, considering the expenses and discomfort associated with each type of procedure. Above all, numerous prospective studies in the past posed serious 
limitations with the small number of patients, significant expenses, and poor methodological qualities (38). In addition, either placebo response or response to steroids and to the injectate can be easily discounted as all these patients underwent all such modalities on multiple occasions and facet joint and sacroiliac joint pain was ruled out as a major component. This study also included improvement in functional status with increased ability to perform activities of daily living and improvement in the activities the patients liked to perform in their daily life instead of return to work criteria. While the physician who evaluated these patients was part of the organization, he was not involved in the care of the patients being studied.

The cost effectiveness evaluation utilized in this study also appears to be optimal, even though it may be criticized. It is well known that outcomes in chronic pain management, though extremely difficult to evaluate, may be assessed by evaluation of quality of life or improvement in functional status, health status, health related quality of life, well being of the patient, satisfaction with care, health services utilization, expenditure analysis, and medical findings (39-42). However, quality of life or improvement in functional status, which evaluates the patient's abilities to function in his or her own world is considered as the most important aspect of evaluation in pain management. Hopwood (39) in discussing quality-adjusted-life-years (QALYs), reported that for pain patients, the trade off may be 1 year of pain for 0.5 years of pain-free status, equaling 0.5 quality-adjusted-life-years. Our cost effectiveness analysis was determined by utilizing the criteria as described above with 1 year of quality-adjusted-life-years. This is similar to the other cost-effectiveness studies conducted in the past. However, this analysis is based on patients treated in an outpatient surgical facility and with physician and facility charges that were reasonable and customary without creative billing.

It is also of significance to note that the previous cost effectiveness study of non-endoscopic adhesiolysis with hypertonic saline neurolysis in the management of low back pain by Manchikanti et al (28) showed an achievement of 1 year of QALYs with a cost of \$5,564 in contrast to the present study with a cost per QALYs of $\$ 2,080$. This discrepancy may be explained in part by heterogenous composition of patients in the previous study which included only $37 \%$ post surgical patients, whereas this study included $100 \%$ of post-surgical patients. In addition, the percent of patients with traumatic onset in this study was $65 \%$, whereas in the previous study, it was less than $40 \%$. Since cost-effectiveness calculations included actual reimbursement, it is conceivable that reimbursement is steadily declining in general and Medicare and Medicaid recipients are also increasing. Finally, it may also be attrib- uted to the refinements in the technique and ability to perform proper adhesiolysis.

The significant differences noted in this study include lower cost with non-endoscopic adhesiolysis compared to endoscopic adhesiolysis. This may be explained partially on a multitude of factors: endoscopic adhesiolysis, in general, is an expensive procedure with expensive equipment leading to higher facility charges; and there is no involvement of separate anesthesiology services with non-endoscopic adhesiolysis in contrast to endoscopic adhesiolysis.

In conclusion, non-endoscopic epidural adhesiolysis and administration of corticosteroids and hypertonic saline is a safe and cost effective procedure for relieving chronic intractable pain in post lumbar laminectomy patients who fail to respond to other modalities of treatment, including other types of neural blockade when performed in an outpatient setting with reasonable and customary charges. Similarly, endoscopic adhesiolysis with the administration of corticosteroids is also a safe and possibly cost-effective technique for relief of chronic intractable pain failing to respond to other modalities of treatments when performed in an outpatient setting. However, cost effectiveness of non-endoscopic adhesiolysis with administration of corticosteroids and hypertonic saline appears to be superior to endoscopic adhesiolysis.

\section{ACKNOWLEDGMENTS}

The author wishes to thank Michelle R. Burkeen for her assistance with transcription of the manuscript and William L. Wade, Professor of English and Bert Fellows, M.A. for review of the manuscript.

\section{REFERENCES}

1. Wilkinson HA. Introduction: Etiology, diagnosis, and therapy. In: The failed back syndrome. Etiology and therapy. Second Edition. New York, Springer-Verlag, 1992;pp1-3.

2. Wilkinson HA. The role of improper surgery in the etiology of the failed back syndrome. In: The failed back syndrome. Etiology and therapy. Second Edition. New York, Springer-Verlag, 1992;pp4-12.

3. Law JD, Lehman RAW, Kirch WM. Reoperation after lumbar intervertebral disc surgery. $J$ Neurosurg 1978;48:259-263.

4. Biondi J, Greenberg BJ. Redecompression and fusion in failed back syndrome patients. J Spinal Disord 1990; 3:362-369.

5. Turner JA, Ersek M, Herron L et al. Surgery for lum- 
bar spinal stenosis, attempted meta-analysis of the literature. Spine 1992;17:1-7.

6. Waddell G, Kummel EG, Lotto WN et al. Failed lumbar disc surgery and repeat surgery following industrial injury. J Bone Joint Surg (Am) 1979;61:201-207.

7. Ross JS, Robertson JT, Frederickson RCA et al. Association between peridural scar and recurrent radicular pain after lumbar discectomy: magnetic resonance evaluation. Neurosurgery 1996;38:855-863.

8. Fritsch EW, Heisel J, Rupp S. The Failed Back Surgery Syndrome. Reasons, intraoperative findings, and long-term results: a report of 182 operative treatments. Spine 1996;21:626-633.

9. Parke WW, Watanable R. Adhesions of the ventral lumbar dura. Adjunct source of discogenic pain? Spine 1990; 15:300-303.

10. Quiles M, Marchisello PJ, Tsairis P. Lumbar adhesive arachnoiditis: Etiological and pathological aspects. Spine 1978; 3:45-50.

11. Benoist M, Ficat C, Baraf P, et al. Post operative lumbar epiduroarachnoiditis: Diagnostic and therapeutic aspects. Spine 1980; 5:432-436.

12. Fager CA, Freidberg SR. Analysis of failures and poor results of lumbar spine surgery. Spine 1980; 5:87-94.

13. Hanley EN, Shapiro DE. The development of low back pain after excision of a lumbar disc. J Bone Joint Surg 1989; 71A:719-721.

14. Rutkow IM. Orthopaedic operations in the United States, 1979-1983. J Bone Joint Surg 1986; 68A:716719.

15. Davis H. Increasing rates of cervical and lumbar spine surgery in the United States, 1979-1990. Spine 1994; 19:1117-1124.

16. Burton CV. Causes of failure of surgery on the lumbar spine: Ten-year follow-up. Mt Sinai J Med 1991; 58:183-187.

17. Burton CV, Kirkaldy-Nillis WH, Yong-Hing K, et al. Causes of failure of surgery on the lumbar spine. Clin Orthop 1981; 157:191-199.

18. North RB, Campbell JN, James CS, et al. Failed back surgery syndrome: 5 year follow-up in 102 patients undergoing repeated operation. Neurosurgery 1991; 28:685-691.

19. Cauchoix J, Ficat C, Girard B. Repeat surgery after disc excision. Spine 1978; 3:256-259.

20. Pawl RP. Arachnoiditis and epidural fibrosis: The relationship to chronic pain. Current Review of Pain 1998; 2:93-99.

21. Cook SD, Prewett AB, Dalton JE, et al. Reduction in perineural scar formation after laminectomy with Polyactive ${ }^{\circledast}$ membrane sheets. Spine 1994; 19:18151825.

22. Racz GB, Heavner JE, Diede JH. Lysis of adhesions utilizing the epidural approach. In: Waldman SD, Winnie AP (eds). Interventional pain management. WB Saunders, Philadelphia. 1996; pp 339-351.

23. Racz GB, Holubec JT. Lysis of adhesions in the epidural space. In: Racz GB, (ed.) Techniques of neurolysis. Boston, Kluwer Academic Publishers, 1989;pp57-72.

24. Racz GB, Sabonghy M, Gintautas J, et al. Intractable pain therapy using a new epidural catheter. JAMA 1982;248:579-581.

25. Racz GB, Haynsworth RF, Lipton S. Experiences with an improved epidural catheter. Pain Clinic 1986;1:2127.

26. Racz GB, Heavner JE, Raj PP. Percutaneous epidural neuroplasty. Prospective one-year follow up. Pain Digest 1999; 9:97-102.

27. Heavner JE, Racz GB, Raj P. Percutaneous epidural neuroplasty. Prospective evaluation of $0.9 \% \mathrm{NaCl}$ versus $10 \% \mathrm{NaCl}$ with or without hyaluronidase. Reg Anesth Pain Med 1999; 24:202-207.

28. Manchikanti L, Pakanati RR, Bakhit CE, et al. Role of adhesiolysis and hypertonic saline neurolysis in management of low back pain. Evaluation of a modification of Racz protocol. Pain Digest 1999; 9:91-96.

29. Saberski LR, Brull S. Fiberoptic visualization of the spinal cord. A historical review and report of current methods. Yale Biol Med 1995:68:7-16.

30. Addison RG. Spinal endoscopy. Current Review of Pain 1999; 3:116-120.

31. Manchikanti L, Pakanati RR, Pampati V. The Value and Safety of Epidural Endoscopic Adhesiolysis. Am J Anesthesiol 1999; in press.

32. Devulder J, Lutgarde B, Castille F, et al. Relevance of epidurography and epidural adhesiolysis in chronic failed back surgery patients. Clin J Pain 1995; 11:147150.

33. Mueller-Schwefe G, Hassenbusch SJ, Reig E. Cost effectiveness of intrathecal therapy for pain. Neuromodulation 1999; 2:77-84.

34. Cicala RS, Wright H. Outpatient Treatment of patients with chronic pain. Analysis of cost savings. Clin J Pain 1989:5:223-226.

35. Malter AD, Larwon EB, Urban N, et al. Cost-effectiveness of lumbar discectomy for the treatment of herniated intervertebral disc. Spine 1996:21:1048-1055.

36. Williams A. Economics of coronary artery bypass grafting. Br Med J 1985:291:325-329.

37. Lave JR, Frank RG, Schulberg HC, et al. Cost-effectiveness of treatments for major depression in primary care practice. Arch Gen Psychiatry 1998:55:645-651.

38. Koes BW, Bouter LM, Van Der Heijden GJMGV. Methodological quality of randomized clinical trials on treatment efficacy in low back pain. Spine 1995:20:228235.

39. Hopwood M. Outcomes assessment in pain management. In: Pain Management. Abram SE, volume editor, Philadelphia, Churchill-Livingston, 1998, pp14.114.11.

40. Davies A, Doyle AT, Lansky D, et al. Outcomes assessment in clinical settings: A consensus statement on principles and best practices in project management. Joint Comm Quality Improve 1994;20(1)6-16.

41. Epstein RS, Sherwood LM. From outcomes research to disease management: A guide for the perplexed. Ann Intern Med 1996:124:832-837.

42. Lynn J, Virnig BA. Assessing the significance of treatment effects: comments from the perspective of ethics. Med Care 1995:33:AS292-AS298. 